\title{
Late Intrathecal Cell Therapy Increases Brain Glucose Metabolism and Improves the Long-Term Established Sequelae of Cerebral Hemorrhage
}

\author{
Sara Fernández-Guinea ${ }^{1}$, Mercedes Zurita², Estefanía García ${ }^{1}$, Jorge Mucientes ${ }^{3}$, \\ Cristina López ${ }^{4}$, Cecilia Fernández-Mateos ${ }^{2}$ and Jesús Vaquero ${ }^{2 *}$ \\ ${ }^{1}$ Department of Experimental Psychology, Complutense University, Madrid, Spain \\ ${ }^{2}$ Service of Neurosurgery, Puerta de Hierro-Majadahonda Hospital, Autonomous University, Madrid, Spain \\ ${ }^{3}$ Service of Nuclear Medicine, Puerta de Hierro-Majadahonda Hospital, Autonomous University, Madrid, Spain \\ ${ }^{4}$ Lescer Center for Neurological Rehabilitation, Madrid, Spain
}

*Corresponding author: Jesús Vaquero, Service of Neurosurgery, Puerta de Hierro-Majadahonda Hospital, Autonomous University, Manuel de Falla, 1, 28222-Madrid, Spain

\begin{abstract}
Background: The possibility of an increase in brain glucose metabolism after intrathecal administrations of autologous mesenchymal stromal cells (MSCs) has been recently reported, suggesting a role in the treatment of neurological disorders. We describe here the utility of this type of cell therapy in chronic neurological sequelae due to cerebral hemorrhage.
\end{abstract}

Methods: A 49-year-old female suffered from 2009 severe neurological sequelae after spontaneous intracerebral hemorrhage open to ventricle in the left frontoparietal region. Eight years later, she received 3 intrathecal administrations of 100 million autologous MSCs each three months, until a total dose of 300 million.

Results: From the first administration of MSCs, the patient reported a clear and progressive improvement that is maintained one year after finishing the cell therapy. The neurological improvement was associated to a progressive increase in global brain glucose metabolism measured by 18F-fluorodeoxyglucose positron emission tomography (18F-FDG-PET).

Conclusions: Late intrathecal administration of cell therapy with autologous MSCs may be a useful strategy to achieve improvement of chronic neurological sequelae due to hemorrhagic stroke.

\section{Keywords}

Cerebral hemorrhage, cell therapy, brain metabolism, stroke
After numerous preclinical studies and a growing experience in patients, there seems to be evidence of the usefulness of cell therapy in stroke, improving functional recovery [1-3]. However, these studies have generally been performed in acute phases, and the benefit has been attributed to the well-known properties of cell therapy, achieving neuroprotective effect or enhancing endogenous neurogenesis. At present, there are few data about the possible effect of cell therapy in late phases after stroke, and its possibility of achieving a long-term modification of the chronic established neurological sequelae.

Taking into account the importance of knowing the therapeutic window in which cell therapy can be useful in the treatment of stroke, in the past our group applied delayed cell therapy with Mesenchymal Stromal Cells (MSCs) after experimental cerebral hemorrhage in rodents, obtaining evidence that intracerebral administration of MSCs two months after brain hemorrhage can improve established neurological sequelae $[4,5]$. We describe here a patient in whom intrathecal administration of autologous MSCs, 8 years after the hemorrhagic stroke, improved established chronic sequelae. In this patient, clinical improvement was associated with a progressive increase in brain glucose metabolism, mea-

Citation: Fernández-Guinea S, Zurita M, García E, Mucientes J, López C, et al. (2018) Late Intrathecal Cell Therapy Increases Brain Glucose Metabolism and Improves the Long-Term Established Sequelae of Cerebral Hemorrhage. Int J Stem Cell Res Ther 5:058. doi.org/10.23937/2469-570X/1410058 Accepted: December 22, 2018: Published: December 24, 2018

Copyright: (c) 2018 Fernández-Guinea S, et al. This is an open-access article distributed under the terms of the Creative Commons Attribution License, which permits unrestricted use, distribution, and reproduction in any medium, provided the original author and source are credited. 
sured by $18 \mathrm{~F}$-fluorodeoxyglucose positron emission tomography (18F-FDG-PET) a finding recently described in patients undergoing this type of cell therapy for sequelae of traumatic brain injury (TBI) [6]. The increase of brain metabolism is a new effect of intrathecal MSCs administration and can offer a new explanation for the efficacy of late cell therapy in neurological disorders.

\section{Case Report}

\section{History}

This 49-year-old female, right-handed, and computer engineer, with more than 20 years of formal education, suffered in 2009 from spontaneous intracerebral hemorrhage in the left frontoparietal area, open to ventricule. Angiographic study did not show presence of aneurysms or vascular malformations. After spontaneous resolution of the hemorrhage, the patient has been receiving a comprehensive neurorehabilitation program of physiotherapy, occupational therapy, speech therapy and neuropsychological intervention. Currently, she continues her physiotherapy and neuropsychological treatment. In 2017, 8 years after brain hemorrhage, the patient comes to us requesting a possible treatment of cell therapy.

\section{Examination}

At this time a study of Magnetic Resonance (MR) showed a large intracerebral cavity in the area where the hemorrhage took place, communicated with the ventricular system (Figure 1), and 18F-FDG-PET showed hypometabolism in the areas adjacent to the lesion, and in posterior region of the left basal ganglia.

Clinical examination showed residual right hemiparesis (great difficulty in gait, loss of balance and instability requiring the use of external supports), and severe spasticity on right upper extremity, especially on hand, with no functionality. She complained of communication problems and cognitive deficits such as memory problems, attention difficulties (mental fatigue after few minutes using the computer, difficulties to follow
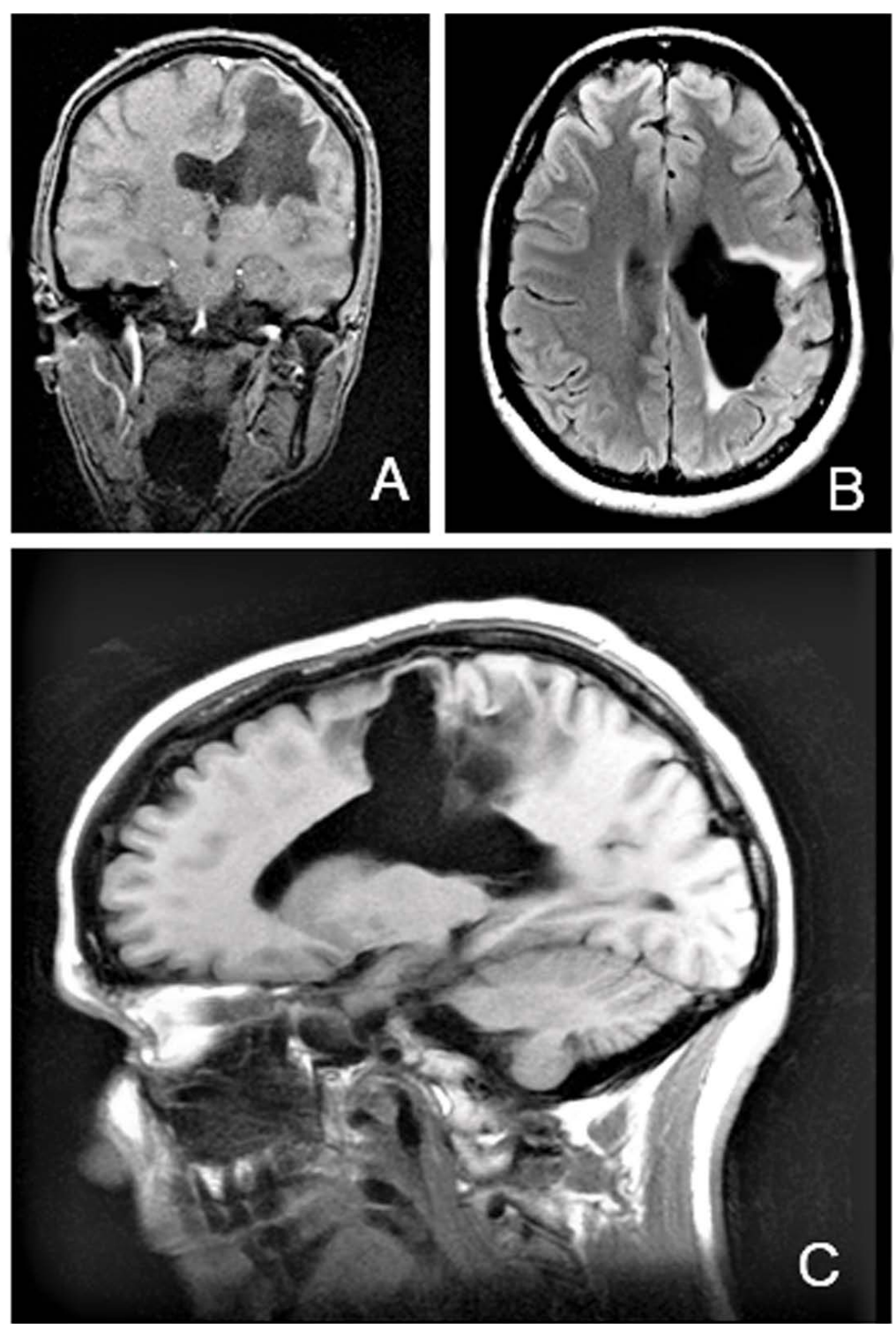

Figure 1: $A, B, C)$ Aspects of Magnetic Resonance (MR) in 2017, showing the residual lesions on the left cerebral hemisphere. 
conversations, to attend several stimuli at the same time), anomia, reading problems (she hardly could read half page without forgetting it or be feeling very tired). Neuropsychological assessment revealed a poor performance, mainly in attention, learning, and memory, planning, naming, verbal fluency, speech and gesture apraxia tasks.

Spanish Agency for Medications and Health Products (AEMPS), and Medical Management of Puerta de Hierro-Majadahonda Hospital authorized us the treatment with our NC1 medicament. It is a cell therapy medicament developed in our clean room of the neurosurgical service of the Puerta de Hierro-Majadahonda Hospital, and currently approved as a medicament under clinical investigation (PEI No. 12-141). It consists of expanded autologous MSCs obtained from bone marrow, and autologous plasma as its excipient. Techniques for culture and expansion of MSCs, phenotypic characterization, formulation and packaging have been previously described [7-9].

\section{Treatment}

Between March 2017 and September 2017, the patient received 3 intrathecal administrations of 100 million de autologous mesenchymal stromal cells each three months, until a total dose of $\mathbf{3 0 0}$ million of MSCs.
This treatment study conducted in accordance with the principles of the Declaration of Helsinki [10] and good clinical practice guidelines [11]. Informed consent was obtained, after explaining the experimental nature of the treatment and our previous experience obtained with intrathecal administration of this type of cell therapy in patients with neurological sequelae due to spinal cord injury (SCI) [8] and traumatic brain injury (TBI) [6].

\section{Results}

From the first administration of cell therapy, the patient reported progressively a generalized and appreciable improvement in her mobility and cognitive deficits. At the end of the treatment, a clear reduction in spasticity and improvement in her stability and gait was observed. She can walk for more than one hour without external supports or supervision of her movements. Attention and planning abilities got better. She can work on her computer for several hours without feeling tired, even when she is doing complex tasks. She also enjoys reading for at least one hour, remembering most of it. Articulation of language and oral expression have also improved, and autonomy for daily activities is also increased. Furthermore, prior to cells transplantation, the patient described taking laxatives and a high-fiber diet for constipation. Just after the first cell doses, she
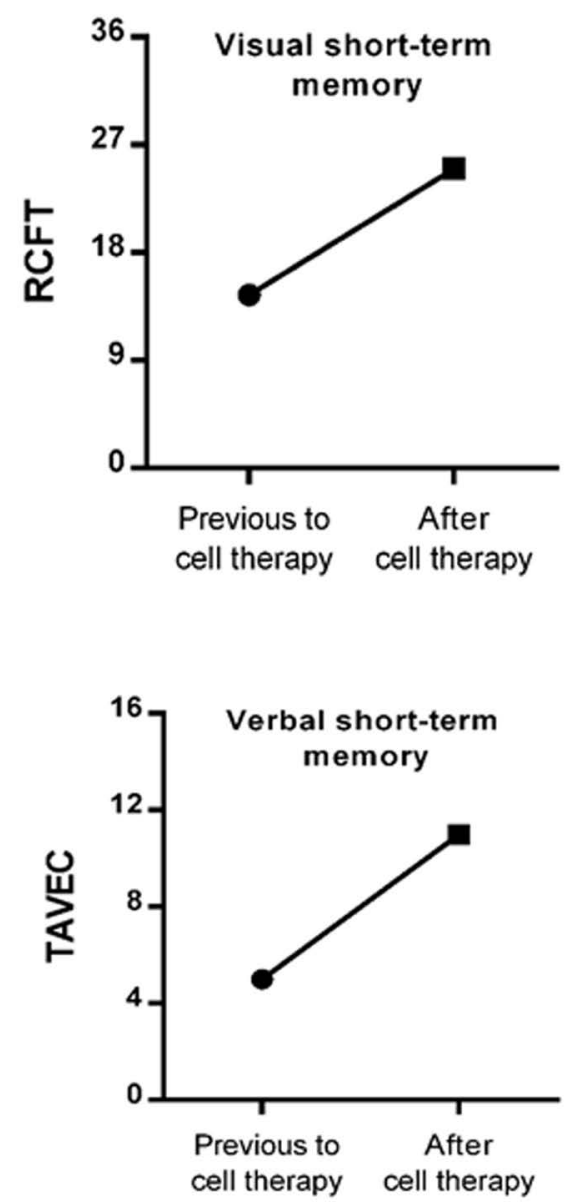
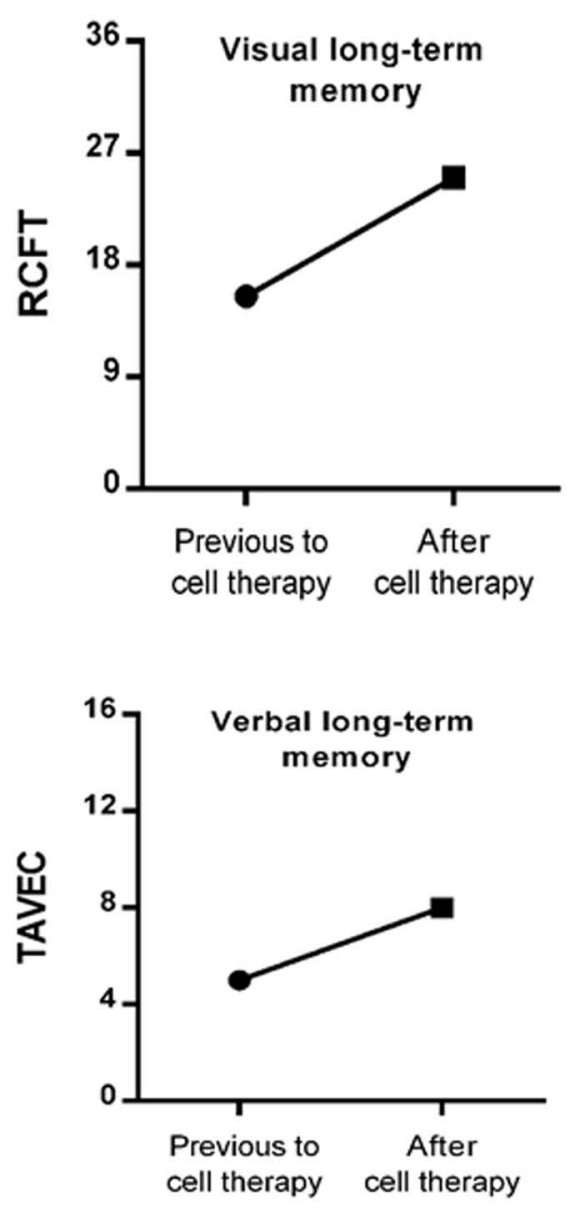

Figure 2: Changes in visual and verbal memory.

RCFT: Rey complex figure test [13]; TAVEC: Spanish adaptation of California verbal learning test [14]. 
experienced no need of them.

Her family, friends, physiotherapist, and neuropsychologist corroborated all these advances.

Neuropsychological assessment confirmed cognitive improvement after cell therapy. She showed a better performance on Addenbrooke Cognitive ExaminationRevised (ACE-R) test [12], with 94 points (79 points previous). Visual and verbal memory, measured by Rey Complex Figure Test (RCFT) [13], and the Spanish adaptation (TAVEC) [14] of California Verbal Learning Test (CVLT) respectively, also increased notably.

Furthermore, the patient had a clear enhancement in planning (Zoo test profile score from -1 to 3) [15], working memory (10 to 12 ) measured by Letters and Numbers test [16], naming 11 to 12 correct spontaneous words, 4 to 3 phonetic cues on Short-Form Boston Naming Test (BNT) [17], and semantic fluency (animals, vegetables, kitchen utensils and clothes), with mean score from 12.25 to 15 . These clinical improvements are maintained one year after finishing MSCs administration. Figure 2 shows changes in visual and verbal memory. Studies with 18F-FDG-PET performed approximately two weeks after each administration of MSCs showed progressive increases in global brain glucose metabolism during the cell therapy (Figure 3).

\section{Discussion}

It is well known, that, in preclinical studies, diverse types of cell therapy can improve neurological sequelae after brain hemorrhage [3-5]. However, the difficulty in maintaining long-term animals models with chronic neurological sequelae after stroke prevents obtaining conclusions about when a possible cell therapy application can be useful. On the other hand, after experimental brain hemorrhage in rodents, neurological improvement has been reported by us after intracerebral transplantation of MSCs, but cell therapy was applied few months after the injury. In these preclinical experiences, the possibllity of a reactivation of endogenous neurogenesis, and cell replacement, has been suggested [4].

Our present case demonstrates, for the first time in patients, that late intrathecal administration of cell therapy with MSCs can improve chronic sequelae due to intracerebral hemorrhage. In our case, after intrathecal administration of MSCs, a progressive increase in cerebral glucose metabolism is observed, suggesting a new mechanism for neurological improvement and
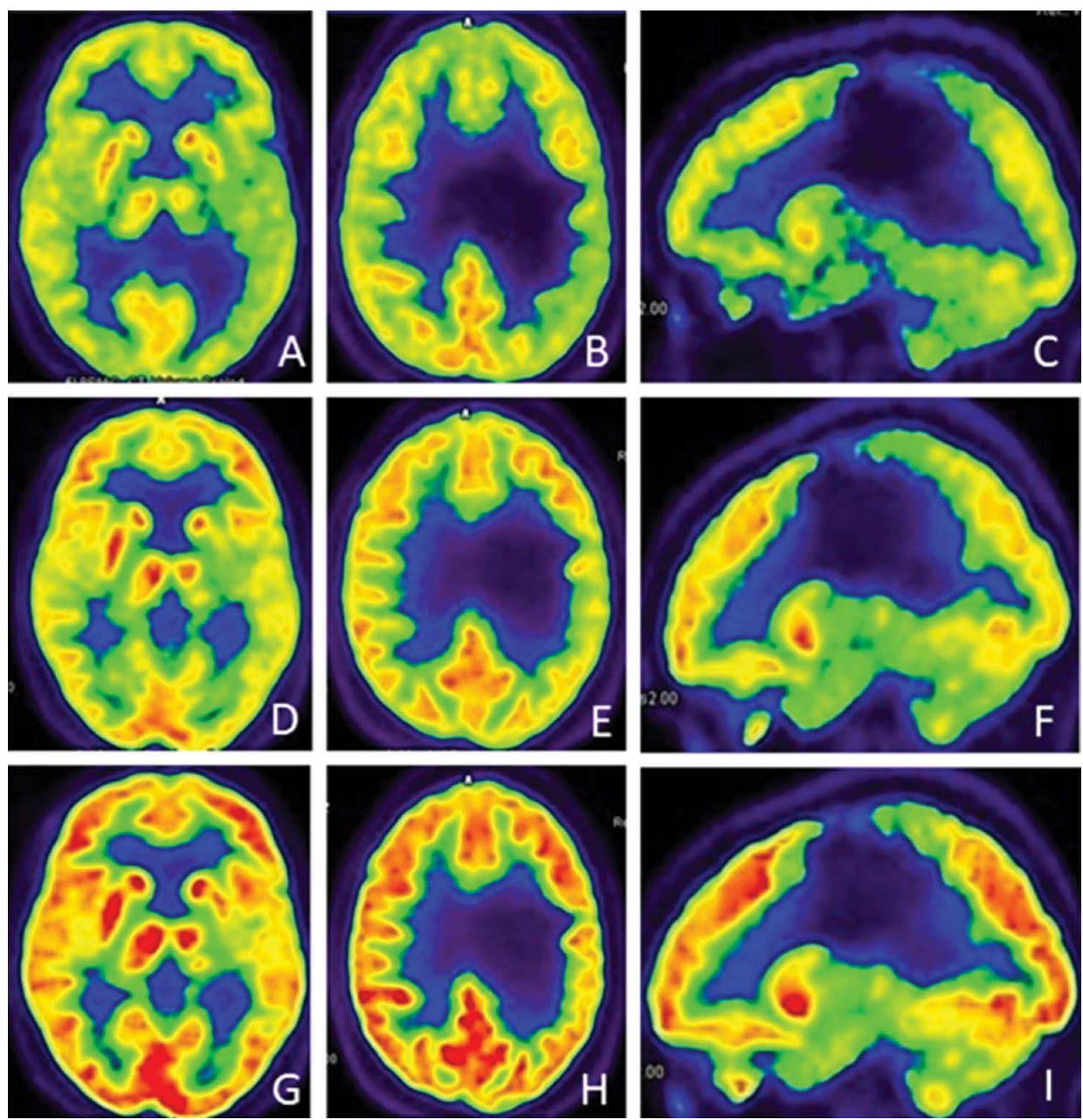

Figure 3: 18F-FDG-PET studies during the cell therapy, showing a progressive increase of brain glucose uptake; $A, B, C)$ After the first intrathecal administration of 100 million autologous MSCs; D,E,F) After the second administration; $G, H, I)$ After the end of cell therapy. 
supports previous findings of our group in patients with severe traumatic brain injury (TBI) [6].

Taking into account the scarce clinical experience in this field of research, we believe it is necessary to study a greater number of patients, in order to confirm the usefulness of intrathecal administration of autologous MSCs in chronic and long-term established sequelae after hemorrhagic stroke. It is obvious that these studies open a new door of hope in patients with lesions until now considered irreversible and a new therapeutic perspective in the field of the contemporary neurology.

\section{Funding Sources}

This study was supported by the Mapfre and Rafael del Pino Foundations.

\section{Conflict of Interests}

None of the authors of this study have any conflicts of interest to disclose.

\section{Acknowledgment}

We thank the institutions supporting our neurological cell therapy program, mainly Mapfre and Rafael del Pino Foundations.

\section{References}

1. Kalladka D, Muir KW (2014) Brain repair: Cell therapy in stroke. Stem Cells Cloning 7: 31-44.

2. Chang Z, Mao G, Sun L, Ao Q, Gu Y, et al. (2016) Cell therapy for cerebral hemorrhage: Five years follow-up report. Exp Ther Med.12: 3535-3540.

3. Gao L, Xu W, Li T, Chen J, Shao A, et al. (2018) Stem cell therapy: A promising therapeutic method for intracerebral hemorrhage. Cell Transplant 1: 963689718773363.

4. Otero L, Zurita M, Bonilla C, Aguayo C, Vela A, et al. (2011) Late transplantation of allogeneic bone marrow stromal cells improves neurological déficits subsequent to intracerebral hemorrhage. Cytotherapy 13: 562-571.

5. Vaquero J, Otero L, Bonilla C, Aguayo C, Rico MA, et al. (2013) Cell therapy with bone marrow stromal cell after intracerebral hemorrhage: impact of platelet-rich plasma scaffolds. Cytotherapy 15: 33-43.
6. Vaquero J, Zurita M, Bonilla $\mathrm{C}$, Fernández $\mathrm{C}$, Rubio $\mathrm{JJ}$, et al. (2017) Progressive increase in brain glucose metabolism after intrathecal administration of autologous mesenchymal stromal cells in patients with diffuse axonal injury. Cytotherapy 19: 88-94.

7. Vaquero J, Zurita M, Rico MA, Bonilla C, Aguayo C, et al. (2016) An approach to personalized cell therapy in chronic complete paraplegia: The Puerta de Hierro phase I/II clinical trial. Cytotherapy 18: 1025-1036.

8. Vaquero J, Zurita M, Rico MA, Bonilla C, Aguayo C, et al. (2017) Repeated subarachnoid administrations of autologous mesenchymal stromal cells supported in autologous plasma improve quality of life in patients suffering incomplete spinal cord injury. Cytotherapy 19: 349-359.

9. Vaquero J, Zurita M, Rico MA, Aguayo C, Bonilla C, et al. (2018) Intrathecal administration of autologous mesenchymal stromal cells for spinal cord injury: Safety and efficacy of the 100/3 guideline. Cytotherapy 20: 806-818.

10. (2000) World medical association declaration of helsinki ethical principles for medical research involving human subjects. JAMA 284: 3043-3045.

11. International conference on harmonisation expert working group (1996) ICH harmonized tripartite guideline. Guideline for good clinical practice.

12. Mioshi E, Dawson K, Mitchell J, Arnold R, Hodges JR (2006) (The Addenbrooke's cognitive examination revised (ACE-R): A brief cognitive test battery for dementia screening. Int J Geriatr Psychiatry 21: 1078-1085.

13. Rey A (1941) L'examen psychologique dans les cas d'encephalopathie traumatique (Les problems). Arch Psychol 28: 215-285.

14. Benedet MJ, Alexandre MA (1998) Test de aprendizaje verbal españa-complutense (TAVEC). Madrid: TEA Ediciones.

15. Wilson BA, Alderman N, Burgess PW, Emslie H, Evans JJ (1996) Behavioural assessment of the dysexecutive síndrome. St Edmunds, UK: Thames valley test company.

16. Wechsler D (1997) Wechsler Memory Scale-Third Edition manual. San Antonio, TX: The Psychological Corporation.

17. Fernandez AL, Fulbright RL (2015) Construct and concurrent validity of the Spanish adaptation of the Boston Naming Test. Appl Neuropsychol Adult 22: 355-362. 